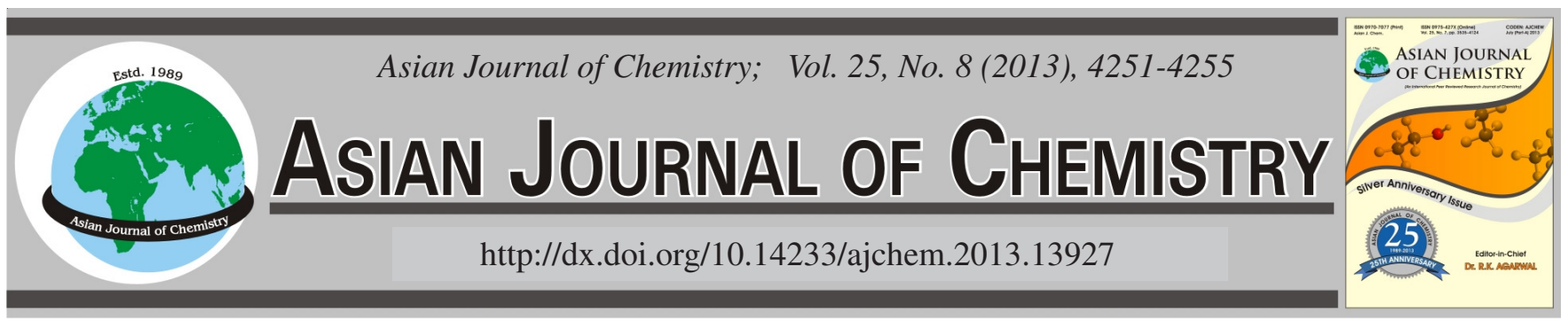

\title{
Gas Chromatographic Separation of Structural Isomers on Cholesteric Liquid Crystal Stationary Phase Materials
}

\author{
YI-Wen LeE ${ }^{1,2}$ and ChiH-Hung LiN ${ }^{3, *}$ \\ ${ }^{1}$ Department of Nursing, National Taiwan University, No. 1, Sec. 4, Roosevelt Road, Taipei, Taiwan \\ ${ }^{2}$ Department of Nursing, Chang Gung University of Science and Technology, 261 Wen-Hwa 1st Road, Kwei-Shan, Tao-Yuan, Taiwan \\ ${ }^{3}$ Center for General Education, Chang Gung University of Science and Technology, 261 Wen-Hwa 1st Road, Kwei-Shan, Tao-Yuan, Taiwan \\ *Corresponding author: Fax: +886 3 2118866; Tel: +886 3 2118999; E-mail: chlin@gw.cgust.edu.tw
}

(Received: 18 April 2012;

Accepted: 7 February 2013)

AJC-12938

\begin{abstract}
A new series of liquid crystalline polysiloxanes with a very wide range of mesomorphic temperatures had been prepared. They contained 4-cholesteryl-(10-undecen-1-yloxy)biphenyl-4'-carboxylate mesogenic and 4-biphenyl-4'-allyloxybenzoate mesogenic side groups displaying an enantiotropic cholesteric phases. The mesomorphic polysiloxane specimen with the widest temperature range was used as the stationary phase in a gas chromatography capillary column and it showed high column efficiency and made great promise for the separation of different structure isomer compounds.
\end{abstract}

Key Words: Isomer, Polysiloxanes, Cholesteric, Liquid crystal, Capillary column.

ᄂ - - - - - - - - - - - - - - - - - - - - - - - - - -

\section{INTRODUCTION}

Liquid crystals had been used as stationary phases ${ }^{1-3}$ in gas chromatography (GC) to separate a variety of compounds including isomeric mixtures which could not be separated on conventional stationary phase. Conventional stationary phase separation of analytes is based on differences in vapour pressures of the solutes and differences in solubility arising from specific energetic interactions. A liquid crystal stationary phase separates analytes based upon differences in solute molecular shape, with the anisotropic packing behaviour of liquid crystalline materials permitting the separation of isomers based on their individual molecular geometries (length-to-breadth) ${ }^{4}$.

Finkelmann and Rehage ${ }^{5}$ were the first to synthesize thermotropic side-chain liquid crystalline polymers. Since then, nematic, smectic and cholesteric liquid crystalline polymers and elastomeric liquid crystalline networks containing a polysiloxane backbone ${ }^{6,7}$ had been synthesized. Polysiloxane had been used as the backbone for the side-chain liquid crystalline polymers because of its properties of low glass transition temperature and high thermostability. Stationary phases based on low molecular weight liquid crystals gain substantial efficiency when they were attached onto flexible polymer backbones. A flexible led group between the backbone and mesogenic unit allowed the resulting polymers to retain liquid crystalline properties. Polymeric stationary phases containing liquid crystalline substituents were desirable for their high thermal stability.
Many liquid crystal polymers of nematic or smectic phase had been reported as stationary phases ${ }^{8,9}$. Cholesteric liquid crystals had also attracted particular interest in their unusual helical supermolecular structure, which could be used as a stationary phase in gas chromatography. Therefore, to take advantage of all these properties and to prepare a gas chromatography stationary phase with optimum separation properties, we selected polysiloxane as the polymer backbone and used an 11-methyl unit as the spacer and modified this backbone with a new series of cholesteric liquid crystalline polymers.

A cholesteric mesophase could be realized by changing the composition of mesogens attached onto a polysiloxane backbone. These materials tended to form cholesteric mesophases over a broad temperature range. For the preparation of novel stationary phases in gas chromatography, we selected the cholesteric mesophase polymers with the widest range of temperatures ${ }^{10,11}$. This study selected nine kinds of isomers, contained four kinds of volatile isomers, these isomers were used for separation on liquid crystal phase column. The cholesteric polysiloxane described herein had merit and had been used to separate isomer compounds successfully.

\section{EXPERIMENTAL}

${ }^{1} \mathrm{H}$ NMR spectra were recorded on a Varian VXR-300 or Bruker $300 \mathrm{MHz}$ spectrometer. Thermal transitions and thermodynamic parameters were determined by using a Seiko SSC/5200 differential scanning calorimeter (DSC) equipped 
with a liquid nitrogen cooling accessory. Heating and cooling rates were $10^{\circ} \mathrm{C} / \mathrm{min}$. Thermal transition reports were collected during the second heating and cooling scans. A SEIKO TG/ DTA 200 thermogravimetric analyzer (TGA) determined thermal decomposition temperatures. A Nikon Microphot-FX optical polarized microscope was equipped with a Mettler FP 82 hot stage and a FP 80 central processor was used to observe the thermal transitions and to analyze the anisotropic textures. Polymerization reactions were traced by using a Nicolet 520 FT-IR.

The gas chromatograph used was always a Hewlett Packard 5890 Series II instrument equipped with a capillary column, split injection system and a FID detector. The carrier gas was $\mathrm{N}_{2}$.

Synthesis of monomers M1-M2: The molecular structure and the general synthetic procedures of these compounds were shown in Schemes I-II and all the final products were examined by the nuclear magnetic resonance spectrometer in order to verify the correction of the molecular structure. The synthesis of monomers 4-biphenyl 4'-allyloxybenzoate (M1) and [S]1-(2-naphthyl)ethyl 6-[4-(10-undecen-1-yl-oxy)biphenyl-4'carbonyloxy]-2-naphthoate (M2) were detailed synthetic procedures for the intermediary compounds and monomers were similar to those reported previously ${ }^{12} .{ }^{1} \mathrm{H}$ NMR chemical shifts of M1 were 4.65(t,2 H,-O- $\left.\mathrm{CH}_{2-}\right) ; 5.48\left(\mathrm{~m}, 2 \mathrm{H},=\mathrm{CH}_{2}\right)$; 6.07(m, 1H, =CH-); 7.03-8.19 (m,13 aromatic protons). ${ }^{1} \mathrm{H}$ NMR chemical shifts of $\mathbf{M} 2$ were 1.24-1.77 (m, $17 \mathrm{H},-\mathrm{CH}_{2-}$ and $\left.-\mathrm{CH}_{3}\right), 1.98\left(\mathrm{~m}, 2 \mathrm{H},=\mathrm{CH}-\mathrm{CH}_{2}-\right), 3.95\left(\mathrm{t}, 2 \mathrm{H},-\mathrm{O}-\mathrm{C}-\mathrm{CH}_{2^{-}}\right.$ $\mathrm{C}=)$, 4.78-4.95 (m, 2H, = $\left.\mathrm{CH}_{2}\right), 5.74(\mathrm{~d}, 1 \mathrm{H},=\mathrm{CH}-), 6.32$ (q, $1 \mathrm{H},-\mathrm{C} * \mathrm{H}-), 6.93-8.62$ (m, 21 aromatic protons).

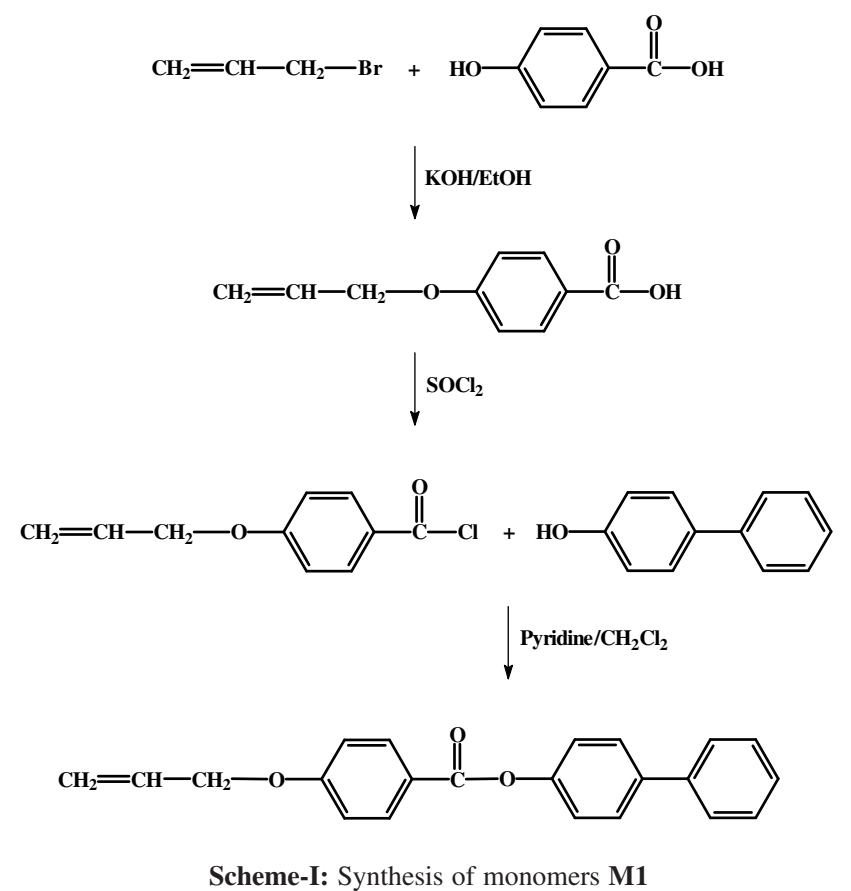

Synthesis of copolysiloxanes P1-P3: All copolysiloxanes P1-P3 were synthesized by the hydrosilylation of poly (methyl hydrogen siloxane) with different ratios of both monomers in the presence of a platinum divinyl tetramethyl disiloxane catalyst. The reaction mixture was refluxed $\left(75^{\circ} \mathrm{C}\right)$ under
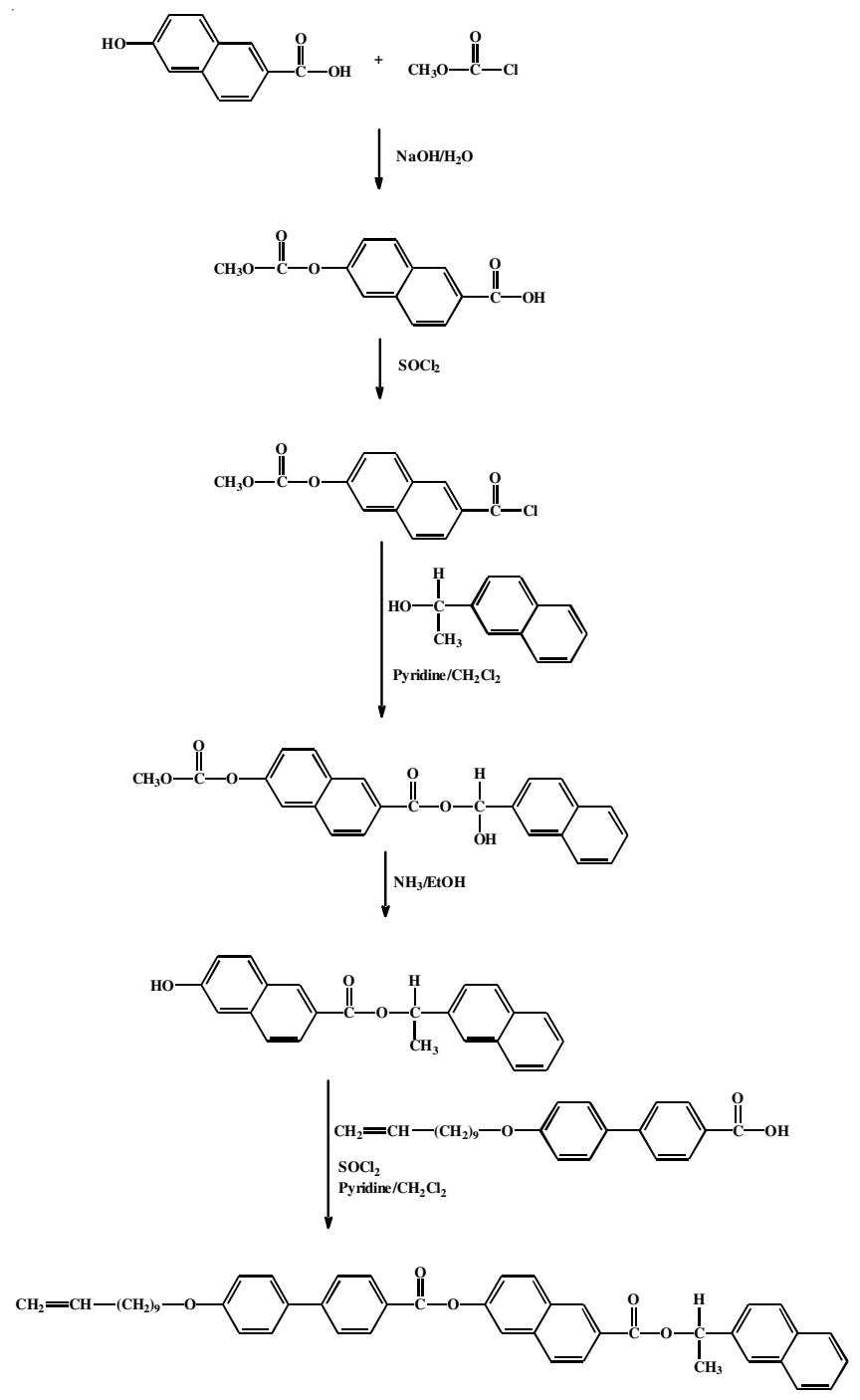

Scheme-II: Synthesis of monomers M2

nitrogen for $2 \mathrm{~h}$. After this reaction time, the FT-IR analysis showed that the hydrosilation reaction was complete. The polymers were separated and purified by several reprecipitations from methylene chloride solution into methanol and then dried under vacuum.

Preparation of the column: A deactivated fused silica capillary column with an I.D. $0.32 \mathrm{~mm} \times 30 \mathrm{~m}$ (Restek) was used. The capillary column was washed with methylene chloride $(20 \mathrm{~mL})$ before coating. The stationary phase $(31.3 \mathrm{mg}$ of copolymer and $1 \mathrm{mg}$ of dicumyl peroxide) was dissolved in methylene chloride $(10 \mathrm{~mL})$. The solution was placed in a rinsing reservoir and forced through the capillary column by $\mathrm{N}_{2}$ gas. After filling, the column was sealed at one end and was placed in a $40^{\circ} \mathrm{C}$ water bath. The column was then placed under vacuum and the solvent evaporated, completing the static coating procedure. The sealed end of the column was opened. The column was cross-linked in a gas chromatography oven, with an oven temperature program of $40-200{ }^{\circ} \mathrm{C}$ at $4{ }^{\circ} \mathrm{C} / \mathrm{min}$ and maintained at $200{ }^{\circ} \mathrm{C}$ for $6 \mathrm{~h}$. The column was cleaned with $10 \mathrm{~mL}$ of methylene dichloride using $\mathrm{N}_{2}$ carrier gas. Finally, the column was installed on a gas chromatography apparatus and conditioned at $200{ }^{\circ} \mathrm{C}$ overnight. 


\section{RESULTS AND DISCUSSION}

We obtained three polymers (P1-P3). The thermal and mesomorphic properties of polymers P1-P3 were measured using differential scanning calorimeter and polarized optical microphotograph. All the synthesized polymers in this study showed liquid crystalline behaviour. The thermal transition temperature and thermodynamic parameter of the polymers were summarized in Table-1. Table-1 summarizes the thermal transitions, ratio and structure of obtained polymers P1-P3, which presented an enantiotropic cholesteric phase. Fig. 1 presented the polarized optical microphotograph of $\mathrm{P} 2$, showed finger print texture of cholesteric phase at $102.3{ }^{\circ} \mathrm{C}$. Polymer P1-P3 exhibited the wide range of temperatures for the cholesteric mesophase. Polymer P3 showed the widest range of temperatures for the cholesteric mesophase, a glass transition at $53.2^{\circ} \mathrm{C}$ and a cholesteric to isotropic phase transition at $234.8^{\circ} \mathrm{C}$ upon DSC heating scan. In the cooling scan, the isotropic to cholesteric phase transition was presented at $225.4{ }^{\circ} \mathrm{C}$. Polymer P3 showed a wide temperature range of the cholesteric phase and the high thermal stability. All of these factors indicated that polymer P3 was well suited to be a gas chromatography stationary phase, thus we selected this new material for all further studies. The capillary column coated with P3 polymer was termed the liquid crystal phase column.

\begin{tabular}{ccc} 
TABLE- 1 \\
& $\begin{array}{c}\text { THERMAL TRANSITIONS AND RATIOS AND } \\
\text { STRUCTURES OF COPOLYMERS }\end{array}$ \\
\hline Polymer & $\begin{array}{c}\text { Monomer feed ratio } \\
\text { M1/M2 }(\mathrm{mol} \%)\end{array}$ & $\begin{array}{c}\text { Phase transitions C, } \\
\text { heating scan cooling scan }\end{array}$ \\
\hline P1 & $10 / 90$ & g 66.0 N*, 196.6 I, I 185 N* \\
P2 & $20 / 80$ & g 54.1 N*, 213.2 I, I 209.4 N* \\
P3 & $30 / 70$ & g 53.2 N*, 234.8 I, I 225.4 N* \\
\hline
\end{tabular}

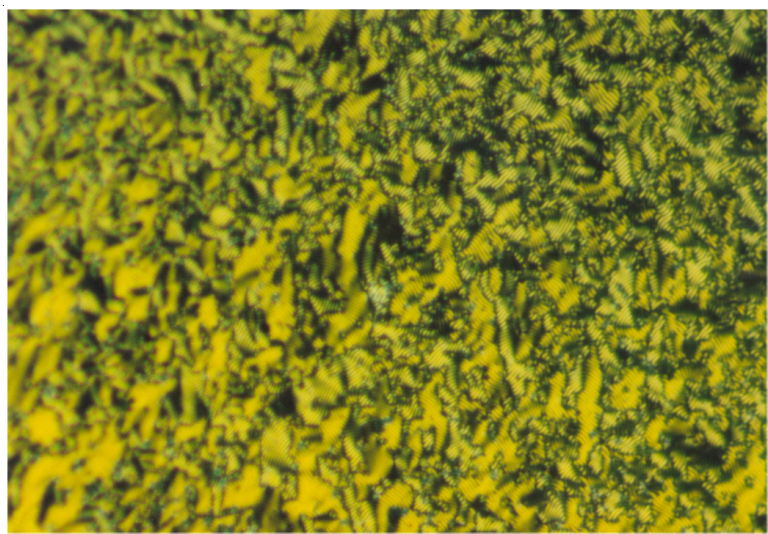

Fig. 1. Typical optical micrograph of polymer P2, finger print texture, $102.3^{\circ} \mathrm{C}$, magnification $200 \mathrm{x}$

The liquid crystal polymer in the cholesteric phase especially had an unusual helical supermolecular structure. This structure showed many similarities to the nematic phase, but every layer showed a twist in the angle. This molecular structure may especially confer on the beneficial effects of the stationary phase for separation of compounds with different length/breadth (L/B) dimensions. This study, selected nine kinds of isomers and contained four kinds of volatile isomers, these isomers were used for separation by liquid crystal phase column. The separation result was listed in Table-2. Most of good separation of the nine kinds of isomers on the liquid crystal phase column was achieved with the exception of $o$-xylene, $m$-xylene, $p$-xylene and thymol and carvacrol. Table-2 contains the accompanying information that summarizes the relative data of the retention time derived from optimized temperature programs, compounds, boiling points and separation factor $(\alpha)$ for the isomers on liquid crystal phase column.

This liquid crystal phase column had the good separation effect on ethylbenzene and $o$-xylene, but $m$-xylene and $p$-xylene were actually difficult to separate, because their boiling points were close $\left(m\right.$-xylene $=139.1^{\circ} \mathrm{C}, p$-xylene $\left.=138.4{ }^{\circ} \mathrm{C}\right)$ and their molecular shapes were very similar. Trans-decalin and cis-decalin isomer compounds had good separation results $(\alpha$ $=1.261$ ), as shown in Fig. 2, because their molecular structures are obviously different.

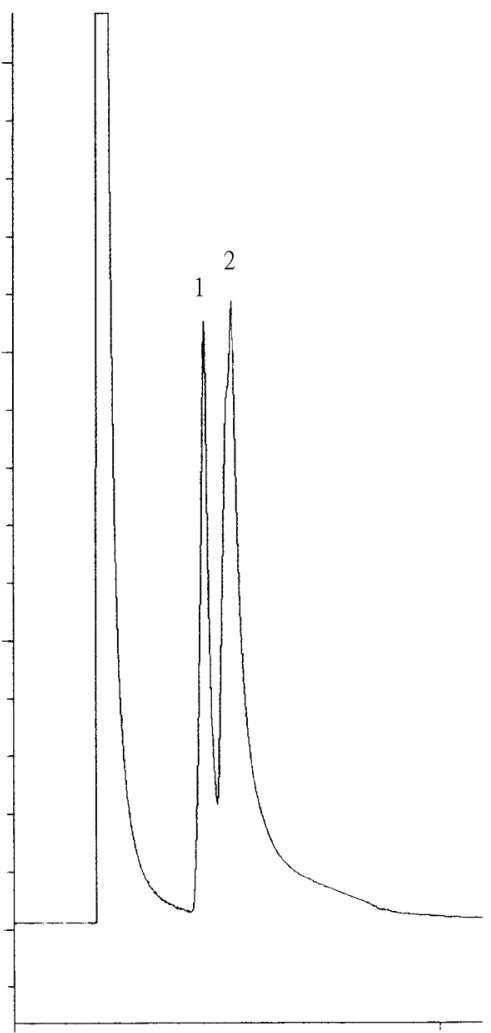

Fig. 2. Chromatogram of the trans-decalin (1), cis-decalin (2) isomers

This study selected three kinds of isomers of polycyclic aromatic compounds. These three kinds of isomers obtained a good separation effect on the LCP column. The isomers of $\mathrm{C}_{14} \mathrm{H}_{10}$ (anthracene and phenanthrene) had a separation factor ( $\alpha=1.225) . \mathrm{C}_{16} \mathrm{H}_{10}$ (fluoranthene and pyrene) isomers had a $\alpha=1.142 . \mathrm{C}_{18} \mathrm{H}_{12}$ (triphenylene, benz[a]-anthracene and chrysene) isomers had a good separation effect. The isomers were difficult to separate by commercial capillary column chromatography ${ }^{13}$. We found that the LCP column showed better resolution for the above isomeric compounds (Table-2). This molecular structure might especially confer on the beneficial effects of the stationary phase for separation of compounds with different $\mathrm{L} / \mathrm{B}$ dimensions. The elution pattern of isomers solutes in this study by the mesomorphic polysiloxane was 
TABLE- 2

RETENTION TIMES OF ISOMERS ON THE LCP COLUMN, THEIR BOILING POINTS, AND THEIR SEPARATION FACTOR

\begin{tabular}{|c|c|c|c|c|}
\hline Compounds & b.p. $\left({ }^{\circ} \mathrm{C}\right)$ & Operate temperature & Retention time (min) & $\alpha$ \\
\hline \multicolumn{5}{|c|}{$\mathrm{C}_{8} \mathrm{H}_{10}$} \\
\hline Ethylbenzene & 136.3 & \multirow{4}{*}{$60{ }^{\circ} \mathrm{C}$ isocratic } & 4.249 & 1.000 \\
\hline$o$-Xylene & 144.4 & & 4.634 & 1.345 \\
\hline$m$-Xylene & 139.1 & & 4.634 & 1.345 \\
\hline$p$-Xylene & 138.4 & & 4.634 & 1.345 \\
\hline \multicolumn{5}{|c|}{$\mathrm{C}_{10} \mathrm{H}_{18}$} \\
\hline trans-Decalin & 187.3 & $30^{\circ} \mathrm{C}$ hold $5 \mathrm{~min}$, then 60 & 5.373 & 1.000 \\
\hline cis-Decalin & 195.7 & ${ }^{\circ} \mathrm{C} / \mathrm{min}$ to $80^{\circ} \mathrm{C}$ & 6.154 & 1.261 \\
\hline \multicolumn{5}{|c|}{$\mathrm{C}_{14} \mathrm{H}_{10}$} \\
\hline Phenanthrene & 340 & \multirow{2}{*}{$170^{\circ} \mathrm{C}$ isocratic } & 6.641 & 1.000 \\
\hline anthracene & 342 & & 7.798 & 1.225 \\
\hline \multicolumn{5}{|c|}{$\mathrm{C}_{16} \mathrm{H}_{10}$} \\
\hline Fluoranthene & 384 & $100^{\circ} \mathrm{C}$ hold $3 \mathrm{~min}$, then 20 & 19.693 & 1.000 \\
\hline pyrene & 404 & ${ }^{\circ} \mathrm{C} / \mathrm{min}$ to $200^{\circ} \mathrm{C}$ & 22.213 & 1.142 \\
\hline \multicolumn{5}{|c|}{$\mathrm{C}_{18} \mathrm{H}_{12}$} \\
\hline Triphenylene & 425 & \multirow{3}{*}{$210^{\circ} \mathrm{C}$ isocratic } & 11.500 & 1.000 \\
\hline Benz[a]-anthrancene & 437.6 & & 12.252 & 1.076 \\
\hline Chrysene & 448 & & 13.764 & 1.230 \\
\hline \multicolumn{5}{|c|}{$\mathrm{C}_{10} \mathrm{H}_{16}$} \\
\hline$\alpha$-Pinene & 155 & $30^{\circ} \mathrm{C}$ hold $1 \mathrm{~min}$, then 20 & 5.145 & 1.000 \\
\hline$\beta$-Pinene & 164 & ${ }^{\circ} \mathrm{C} / \min$ to $120^{\circ} \mathrm{C}$ & 5.530 & 1.446 \\
\hline \multicolumn{5}{|c|}{$\mathrm{C}_{10} \mathrm{H}_{14} \mathrm{O}$} \\
\hline Carvone & 230 & \multirow{3}{*}{$\begin{array}{l}40^{\circ} \mathrm{C} \text { hold } 1 \mathrm{~min} \text {, then } 20 \\
{ }^{\circ} \mathrm{C} / \mathrm{min} \text { to } 100^{\circ} \mathrm{C}\end{array}$} & 10.212 & 1.000 \\
\hline Thymol & 233 & & 10.212 & 1.423 \\
\hline Carvacrol & 237 & & 13.504 & 1.423 \\
\hline \multicolumn{5}{|c|}{$\mathrm{C}_{10} \mathrm{H}_{18} \mathrm{O}$} \\
\hline Eucalyptol & 174 & \multirow{3}{*}{$\begin{array}{l}30^{\circ} \mathrm{C} \text { hold } 1 \text { min, then } 20 \\
{ }^{\circ} \mathrm{C} / \mathrm{min} \text { to } 120^{\circ} \mathrm{C}\end{array}$} & 3.894 & 1.000 \\
\hline Borneol & 206 & & 6.629 & 2.796 \\
\hline Geraniol & 229 & & 8.409 & 3.963 \\
\hline \multicolumn{5}{|c|}{$\mathrm{C}_{10} \mathrm{H}_{12} \mathrm{O}_{2}$} \\
\hline Eugenol & 255 & $40^{\circ} \mathrm{C}$ hold $5 \mathrm{~min}$, then 20 & 5.681 & 1.000 \\
\hline cis-Isoeugenol & 266 & ${ }^{\circ} \mathrm{C} / \mathrm{min}$ to $100^{\circ} \mathrm{C}$ & 7.483 & 1.546 \\
\hline
\end{tabular}

consistent with the degree of their rod-like geometry, with the more rod-like structures being retained longer. For example, phenanthrene $(\mathrm{L} / \mathrm{B}=1.459)$ eluted earlier than anthracene $(\mathrm{L} /$ $\mathrm{B}=1.559)$, the retention time of fluoranthene $(\mathrm{L} / \mathrm{B}=1.142$, b.p. $=384^{\circ} \mathrm{C}$ ) was relatively short, because boiling point of fluoranthene was lower by $20^{\circ} \mathrm{C}$ than pyrene $(\mathrm{L} / \mathrm{B}=1.119$, b.p. $\left.=404{ }^{\circ} \mathrm{C}\right)$. The isomers of triphenylene $(\mathrm{L} / \mathrm{B}=1.120)$, benz $[\mathrm{a}]$-anthracene $(\mathrm{L} / \mathrm{B}=1.580)$ and chrysene $(\mathrm{L} / \mathrm{B}=1.720)$ had the good separation effect on LCP column (Fig. 3). When the separation of retention time of the triphenylene was shortest, the benz[a]anthracene was the best and chrysene was the longest. The result and the molecular length and breadth were compared to related values, when length and breadth were compared to the big value, the retention time would be longer.

In this study, we selected four kinds of volatile isomers. These four kinds of isomers obtained a good separation effect on LCP column. The isomers of $\mathrm{C}_{10} \mathrm{H}_{16}(\alpha$-pinene, $\beta$-pinene) had a good separation effect on LCP column $(\alpha=1.446)$. Although they had a little different boiling points ( $\alpha$-pinene, b.p. $=155^{\circ} \mathrm{C} ; \beta$-pinene, b.p. $\left.=164{ }^{\circ} \mathrm{C}\right)$ but their structures had different duplet bond positions ( $\alpha$-pinene's duplet bond is in six ring; $\beta$-pinene's duplet bond is outside six ring).

$\mathrm{C}_{10} \mathrm{H}_{14} \mathrm{O}$ (carvone, thymol, carvacrol) isomers, carvone had the good separation effect, thymol and carvacrol's separation effect were not good. Because the separation effect was

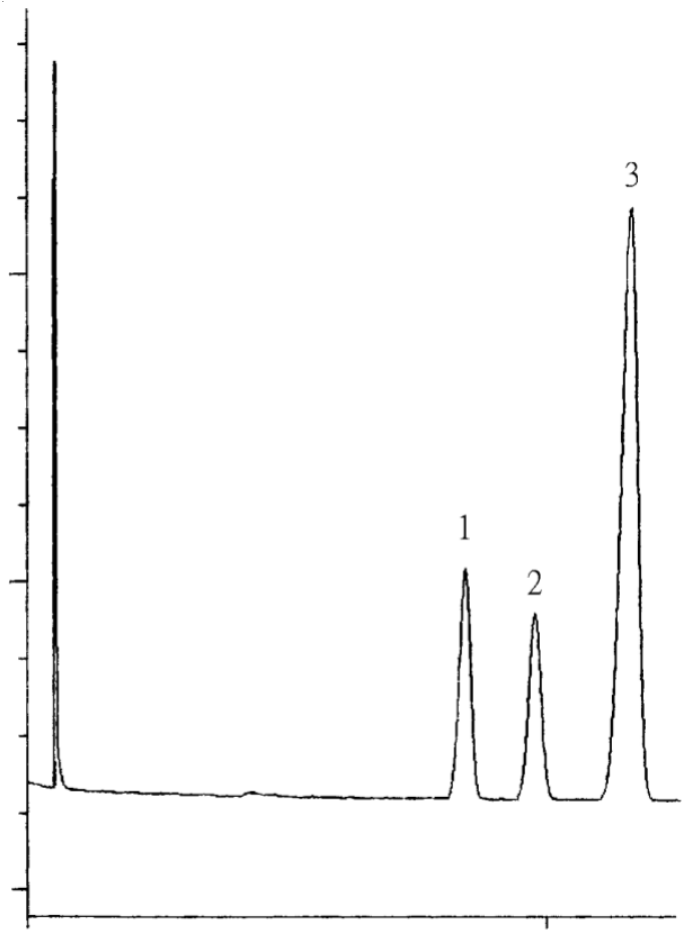

Fig. 3. Chromatogram of the triphenylene (1), benz[a]anthracene (2), chrysene (3) isomers 
influenced by the compound's boiling point and the shape of molecule, these isomers had slightly different boil points (carvone b.p. $=230{ }^{\circ} \mathrm{C}$, thymol b.p. $=233{ }^{\circ} \mathrm{C}$, carvacrol b.p. $=$ $237^{\circ} \mathrm{C}$ ) and they had different structure arrangement. Carvone molecular structure belonged to the non-plane six-ring structure, but thymol and carvacrol belonged to the planar molecule structures. Therefore, carvone compound had shorter retention time than thymol and carvacrol did. Thymol and carvacrol could not separate on LCP column.

In Fig. 4, eucalyptol, borneol and geraniol $\left(\mathrm{C}_{10} \mathrm{H}_{18} \mathrm{O}\right)$ isomers had the good separation effect on LCP column. Eucalyptol molecular structure was six-membered-ring and included a bridge, which was a carbon and an oxygen atom composition, therefore its' structure is like a spherical. Borneol molecular structure was six-membered-ring and had a bridge,

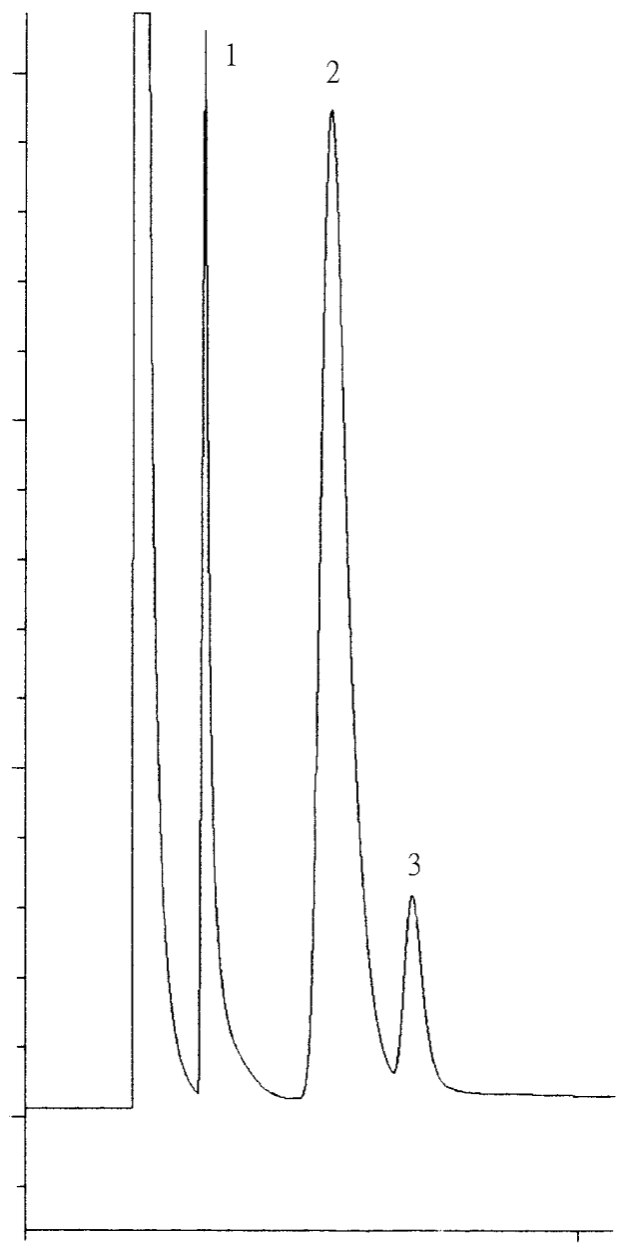

Fig. 4. Chromatogram of the eucalyptol (1), borneol (2), geraniol (3) isomers which was a carbon composition, its' structure is like a spheroid. Geraniol molecular structure was a straight chain molecule and its' structure is like clavate molecule. Liquid crystal compound as stationary phase materials in GC, especially had the good separation effect on the shapes of different isomers. The retention time of the clavate-shaped type of the molecule would be longer than the retention time of the spherical type of the molecule, therefore eucalyptol was eluted earlier than borneol was and borneol was eluted earlier than geraniol was. $\mathrm{C}_{10} \mathrm{H}_{12} \mathrm{O}_{2}$ (eugenol, cis-isoeugenol) isomers had a good separation effect on LCP column $(\alpha=1.546)$. Thus, the LCP column showed the good resolution of nine kinds of isomers.

\section{Conclusion}

The side-chain liquid crystalline polysiloxanes with wide temperature ranges of cholesteric nematic phase had been proven to be useful in separating many classes of compounds. The obtained polymers showed high thermal stability and had wide temperature ranges associated with the cholesteric liquid crystal phase. These results might be shown due to the twisted packing structure of the cholesteric mesophase exhibited by the new stationary phases. Because the separation was based on the molecular shape, isomers that had very similar intrinsic properties could be separated by these kinds of mesomorphic polymer stationary phases. The prepared column described here displayed high column efficiency and made great promise for the separation of a wide range of isomer compounds.

\section{REFERENCES}

1. Y.W. Lee and C.H. Lin, Asian J. Chem., 24, 5190 (2012).

2. H. Kelker, Fresenius Z. Anal. Chem., 198, 254 (1963).

3. W.S. Lee and G.P. Chang-Chien, Anal. Chem., 70, 4094 (1998).

4. A. Radecki, H. Lamparczyk and R. Kaliszan, Chromatographia, 12, 595 (1979).

5. H. Finkelmann and G. Rehage, Makromol. Chem., Rapid Commun., 1, 31 (1980).

6. H. Finkelmann, H.J. Kock and G. Rehage, Makromol. Chem., Rapid Commun., 2, 317 (1981)

7. H. Finkelmann and G. Rehage, Makromol. Chem. Rapid Commun., 3, 859 (1982).

8. G.M. Janin, Adv. Chromatogr., 17, 231 (1979).

9. Z. Witkiewicz, J. Chromatogr. A, 251, 311 (1982).

10. E.G. Bellomo, P. Davidson, M. Imperor-Clerc and T.J. Deming, J. Am. Chem. Soc., 126, 9101 (2004).

11. K. Maeda, Y. Takeyama, K. Sakajiri and E. Yoshima, J. Am. Chem. Soc., 126, 16284 (2004).

12. C.H. Lin and C.S. Hsu, J. Polym. Res., 7, 167 (2000).

13. R.C. Kong, S.M. Fields, W.P. Jackson and M.L. Lee, J. Chromatogr. A, 289, 105 (1984). 